Original Paper (Invited)

\title{
Two-Way Coupled Fluid Structure Interaction Simulation of a Propeller Turbine
}

\author{
Hannes Schmucker ${ }^{1}$, Felix Flemming ${ }^{2}$ and Stuart Coulson ${ }^{2}$ \\ ${ }^{1}$ Voith Hydro GmbH \& Co. KG, Germany \\ Alexanderstraße 11, 89522 Heidenheim, Germany, Hannes.Schmucker@voith.com \\ ${ }^{2}$ Voith Hydro Inc. York, PA, USA \\ 760 East Berlin Road, York, PA, 17408, USA, Felix.Flemming@voith.com, Stuart.Coulson@voith.com
}

\begin{abstract}
During the operation of a hydro turbine the fluid mechanical pressure loading on the turbine blades provides the driving torque on the turbine shaft. This fluid loading results in a structural load on the component which in turn causes the turbine blade to deflect. Classically, these mechanical stresses and deflections are calculated by means of finite element analysis (FEA) which applies the pressure distribution on the blade surface calculated by computational fluid dynamics (CFD) as a major boundary condition. Such an approach can be seen as a one-way coupled simulation of the fluid structure interaction (FSI) problem. In this analysis the reverse influence of the deformation on the fluid is generally neglected. Especially in axial machines the blade deformation can result in a significant impact on the turbine performance. The present paper analyzes this influence by means of fully two-way coupled FSI simulations of a propeller turbine utilizing two different approaches. The configuration has been simulated by coupling the two commercial solvers ANSYS CFX for the fluid mechanical simulation with ANSYS Classic for the structure mechanical simulation. A detailed comparison of the results for various blade stiffness by means of changing Young's Modulus are presented. The influence of the blade deformation on the runner discharge and performance will be discussed and shows for the configuration investigated no significant influence under normal structural conditions. This study also highlights that a two-way coupled fluid structure interaction simulation of a real engineering configuration is still a challenging task for today's commercially available simulation tools.
\end{abstract}

Keywords: Two-way coupling, fluid structure interaction, propeller turbine, CFD, FEA, FSI, gap vortex

\section{Introduction}

The fundamental force on a hydro turbine generating the required shaft torque to drive the generator is the fluid mechanical pressure loading on the turbine blades. This pressure load results in the main structural load on the turbine blade and its connection to the shaft. In an effort to improve performance of hydro turbines while at the same time minimizing the cost of manufacturing the design usually strives for thinner blades. Obviously, the mechanical design then becomes challenging since higher stress levels need to be accounted for, thus a more accurate simulation using finite element analysis (FEA) is essential. At the same time these thinner blades can result in larger deflections of the blade itself due to the lower stiffness of the thinner structure. These deflections therefore change the hydraulic shape of the turbine blade and thus the performance of the hydraulic machine, which in turn has an influence on the pressure loading itself. Especially for low head turbines like bulb, propeller or Kaplan units these small deviations from the designed blade shape can have a significant influence due to the changed velocity discharge profiles into the draft tube.

The current state-of-the-art approach to determine the mechanical stress and deflection of a turbine blade consists of a steady state computational fluid dynamics (CFD) simulation which provides the fluid pressure on the turbine blade. This is then applied as a boundary or load condition for the FEA simulation of the configuration [1]. Such an approach is a so called one-way coupled simulation since no deflection is fed back into CFD. To capture the influence of the deformed blade on the hydraulic performance the deformation has to be brought back into the CFD solution such that an improved solution can be found and the loop can be closed. This comprises a so called two-way coupled fluid structure interaction (FSI) simulation or multi-field simulation and will be discussed and presented in the following sections in detail. In the past, numerous studies have been performed on this FSI simulation strategy applying commercial tools to investigate generic test cases [2] and study the applicability and handling of the tools. Nevertheless, 
applying this simulation strategy to a real propeller turbine blade in an engineering environment was very challenging and highlighted the shortfalls of the available tools. This will be discussed in more detail at the end of the paper.

\section{Multi-Field Simulations}

The term of multi-field simulations arises from the two different problems involved in the FSI problem described in the introduction. On the one hand the water is the fluid field and results in the load on the solid structure and is thereby a driving force. The solid structure on the other hand is the solid field that reacts by means of stresses and deflections to the fluid field. Additionally the resulting deflection of the solid field has an impact on the fluid field that has to adapt to the modified boundary. Finally, if one considers unsteady effects the fluid field not only acts as a driving force, but also as a damping influence on the solid field since fluid mass has to be displaced during the motion of the structure which requires additional energy [2], [3], [4].

The solution of such a multi-field simulation usually requires two separate solvers, one for the fluid (CFD) and one for the structure (FEA) that run in sequential order with synchronization points to exchange information at the interface, namely the turbine blade. In the present work the two commercially available solvers ANSYS CFX 11.0 and ANSYS Classic 11.0 have been applied as CFD and FEA solver respectively. Both have different meshing requirements, therefore different meshes are generated for the fluid field and the solid field. The mesh must not be identical at the interface, but must consist of the identical geometrical surface. In Fig. 1 the simulation strategy utilized by the two ANSYS solvers is demonstrated. At each synchronization point (SP) information is exchanged between the CFD and FEA solution. While CFD provides the forces, FEA returns the resulting deflections and the two meshes are adapted to the new geometry. The three loops reflect the time step loop (X1), the stagger iteration (X2) and the CFD iterations (X3). The complete simulation is running until a convergence criterion is reached, e.g. the change in deflection from iteration to iteration is less than a certain value in the case of a steady state simulation or the selected real time duration is reached in the case of a transient FSI run. Details can be found in the documentation provided by ANSYS [5].

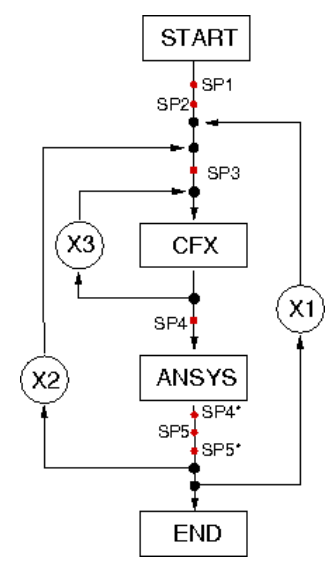

Fig. 1 Flowchart for multi-field simulations with the ANSYS software.

\section{Configuration and Setup}

As a first step, the above described FSI approach has been tested on a generic test case of a wing in between two walls. On the one side it was attached to the wall while on the other side a small gap remained open. This case resembled the general features of the propeller turbine configuration but was simple enough to test the mesh deformation and determine the proper settings of the ANSYS solvers. For brevity, the results are not included in this paper.

The final configuration consists of the AxialT propeller turbine that has been extensively tested in the Consortium on Hydraulic Machines at the Laboratoire de Machines Hydraulic (LAMH) at Laval University by means of advanced laser measurement techniques. The AxialT turbine has been selected for this work due to the velocity measurements of the runner discharge [6] and due to previous studies of this configuration at Voith Hydro [1] even though the turbine model is not a thin blade design and therefore will not exhibit strong deflections. The characteristic of the thickness distribution of the propeller blade is shown in Fig. 2. The values are normalized by the maximum thickness of the blade, which is located at the trunnion of the runner. The values of line2 show the behavior of the blade thickness in the area of maximum thickness. The blade has a large thickness on the hub side, which is $96 \%$ of the maximum thickness, and a continous reducing thickness in radial direction with a minimum thickness of $46 \%$. The thickness also decreases in direction of leading and trailing edge. The minimum thickness of line1, which is located in the leading edge area, has a value of $32 \%$. The trailing edge is specified by a thickness of 17 to $12 \%$ of the maximum thickness of the propeller blade. So the AxialT propeller is characterized by a thick leading edge area and a comparative thick trailing edge compared to new state-of-the-art propeller and Kaplan designs, nevertheless the advantage of the previous investigations were an excellent starting point for basic investigations of fully-coupled numerical simulations. 

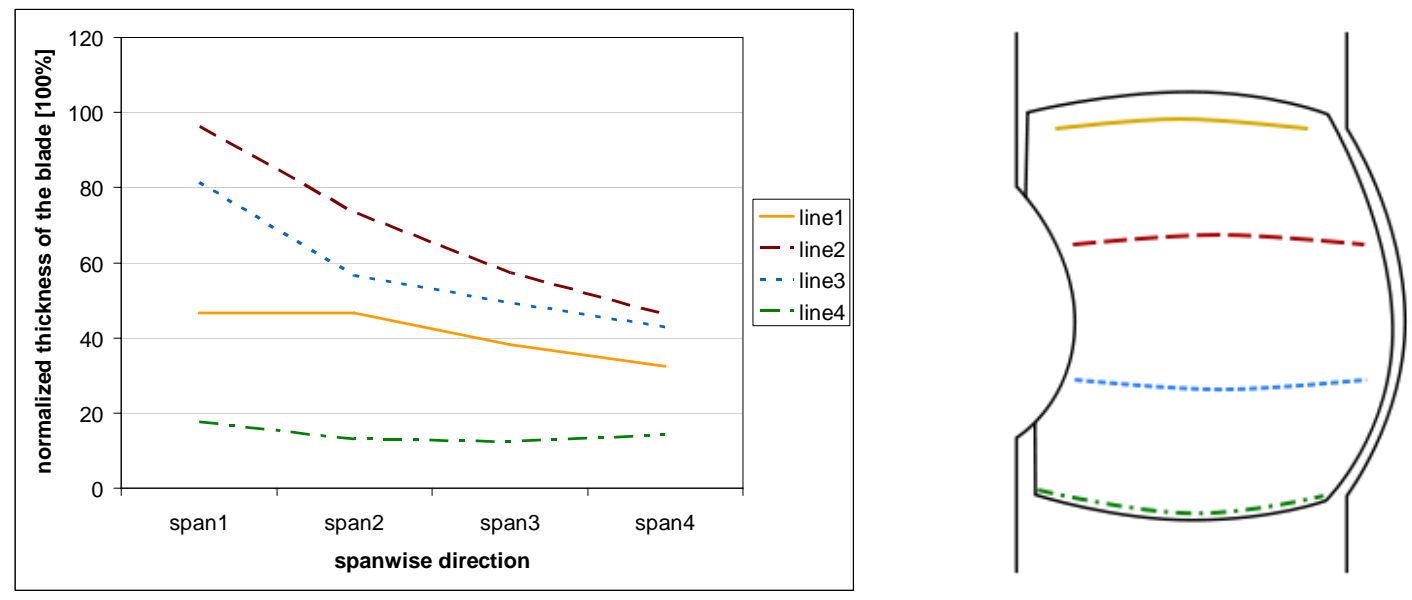

Fig. 2 Thickness allocation of the AxialT propeller at different positions on the blade normalized by the maximum thickness of the blade

The so called maximum power point at a flow rate of $107 \%$ of the optimum flow rate was used for the simulations since the deflections are to be expected highest under full load conditions. Utilizing cyclic periodic boundary conditions one flow channel of the tandem cascade and the runner channel as shown in Fig. 3 were simulated by means of steady state CFD applying a stage interface between the stationary and rotating domain as well as the SST model for turbulence modeling. A nominal gap clearance of 0.3 [mm] was included in the numerical model. The complete model for the fluid mechanical and structural simulation was in model size to allow for a direct comparison to model test results from the laboratory. It is important to note that the standard Voith Hydro CFD tools and meshes were applied in this study to test the feasibility of two-way coupled FSI simulations in an engineering environment. Details on the CFD setup can be found in [1].

The fundamental material properties of the AxialT model turbine blade have been approximated using a Young's Modulus of $117 \cdot 10^{9}\left[\mathrm{~N} / \mathrm{m}^{2}\right]$, a Poisson's Ratio of 0.3 and a density of $8920\left[\mathrm{~kg} / \mathrm{m}^{3}\right]$. These material properties result in the nominal blade stiffness. As mentioned above, the stiffness of the blade is not as low as it could be on a modern low head turbine design due to the increased thickness of the AxialT model blade. Therefore Young's Modulus has been varied to simulate different blade stiffness. Table 1 summarizes all configurations that have been investigated for this study and results will be presented later on. Four different types of simulations were performed and will be described in the following. As future reference, the nominal stiffness configurations are F2, G2, C2a and C2b.

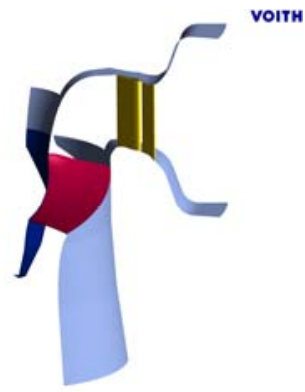

(a)

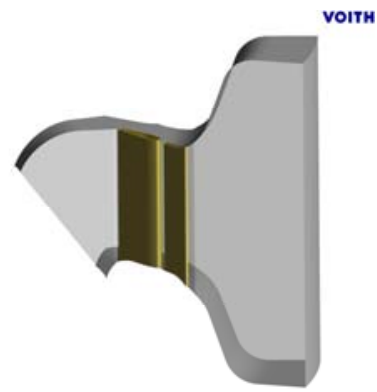

(b)

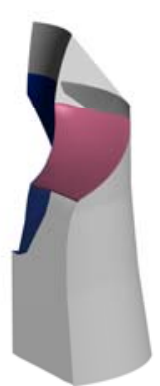

(c) volrs

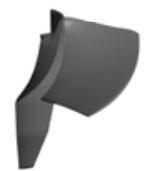

(d)

Fig. 3 AxialT propeller turbine components included in the various CFD (a) - (c) and FEA (d) simulations.

The cases F1 through F4 correspond to fully two-way coupled FSI calculations with a multi-field solution strategy as described in the previous section. Case F1 tries to model a non deformable blade in order to be able to compare a FSI simulation to a pure CFD calculation without any blade deflection. The cases F4 and F4b correspond to the least stiff FSI configuration and are the limiting cases. For a less stiff blade the FSI simulation would fail due to the large displacement of the blade tip and a no longer proper mesh deformation during the simulation. The only difference between F4 and F4b is the resolution of the structural model. While F4 utilized the standard FEA mesh of this study consisting of 13600 elements, the mesh for F4b was refined to 30000 elements in order to check for any mesh dependence of the structural solution. The cases G2 and G4 are also FSI simulations similar to F2 and F4, but this time the peripheral blade tip gap was not included in the simulation.

The cases C1 through C4 are divided into two sub sets. The runs C1a and C2a are pure CFD calculations with an undeformed blade and with a deformed but stiff blade respectively. The deformations in this case were determined by the classical FEA approach using the pressure loads of C1a as load condition (see also [1]). Afterwards, the deformed blade shape has been exported out of ANSYS Classic and a "new" blade was generated and remeshed according to these deflections. One could look at this as a simplified two-way coupled FSI solution that determines the influence of the deformed blade on the flow field. Finally, cases C1b through C4b are pure CFD calculations but based on the geometry and mesh of the converged FSI calculations F1 through F4. These were included to cross check the fluid results of the FSI analysis with the two coupled solvers against a simulation with only the fluid solver. 
Table 1 Overview of different configurations and setups that have been analyzed.

\begin{tabular}{|c|c|c|c|c|c|c|}
\hline \hline Simulation & Young's Modulus & Shroud Gap & Coupling & Solution & Comment & Color \\
\hline F1 & $117 \cdot 10^{20}\left[\mathrm{~N} / \mathrm{m}^{2}\right]$ & yes & 2 way & simultaneous & \\
\hline F2 & $117 \cdot 10^{9}\left[\mathrm{~N} / \mathrm{m}^{2}\right]$ & yes & 2 way & simultaneous & \\
\hline F3 & $117 \cdot 10^{8}\left[\mathrm{~N} / \mathrm{m}^{2}\right]$ & yes & 2 way & simultaneous & \\
\hline F4 & $517 \cdot 10^{7}\left[\mathrm{~N} / \mathrm{m}^{2}\right]$ & yes & 2 way & simultaneous & \\
\hline F4b & $517 \cdot 10^{7}\left[\mathrm{~N} / \mathrm{m}^{2}\right]$ & yes & 2 way & simultaneous & refined structural mesh \\
\hline G2 & $117 \cdot 10^{9}\left[\mathrm{~N} / \mathrm{m}^{2}\right]$ & no & 2 way & simultaneous & \\
\hline G4 & $517 \cdot 10^{7}\left[\mathrm{~N} / \mathrm{m}^{2}\right]$ & no & 2 way & simultaneous & \\
\hline C1a & infinity & yes & none & only CFD & pressure loading for FEA & \\
\hline C2a & "117.10 $\left[\mathrm{N} / \mathrm{m}^{2}\right] "$ & yes & 1 way & separate CFD & regenerated deformed blade & \\
\hline C1b & $117 \cdot 10^{20}\left[\mathrm{~N} / \mathrm{m}^{2}\right]$ & yes & "2 way" & separate CFD & deformed blade from FSI (F1) & \\
\hline C2b & $117 \cdot 10^{9}\left[\mathrm{~N} / \mathrm{m}^{2}\right]$ & yes & "2 way" & separate CFD & deformed blade from FSI (F2) & \\
\hline C3b & $117 \cdot 10^{8}\left[\mathrm{~N} / \mathrm{m}^{2}\right]$ & yes & "2 way" & separate CFD & deformed blade from FSI (F3) & \\
\hline C4b & $517 \cdot 10^{7}\left[\mathrm{~N} / \mathrm{m}^{2}\right]$ & yes & "2 way" & separate CFD & deformed blade from FSI (F4) & \\
\hline
\end{tabular}

\section{Results}

In the following the results of the different simulations will be discussed and compared to each other as well as experimental data. First, the blade deflections and their impact on the pressure load are analyzed. Second, the influence of the blade deflection on the velocity discharge profile into the draft tube is shown and then velocity plots of laser measurements and deformed blades are analyzed. Finally, the resulting shaft torque is compared in detail.

\subsection{Deflection}

The stiffer blade deflects less, as one can see easily from the visualized normalized deflection in Fig. 4. While the nominal configuration (F2) deflects only up to $10 \%$ of the trailing edge thickness (TET) downwards, the least stiff configuration (F4) shows a deflection of $220 \%$, which is significant. The maximum deflection occurs as expected at the tip of the blade and especially towards the trailing edge. Due to the model design, the blade itself is only attached to the hub in the center, approximately for $1 / 3$ of the blade length. The remaining portions towards the leading and trailing edge are covered, so no actual gap is open for the fluid, but no structural support is available. This was included into the structural model and results in the strong deflections towards the trailing edge. It can be assumed that the leading edge deflection will have the biggest influence on the pressure field and possibly leading edge cavitation behavior, while the trailing edge mostly influences the velocity discharge profile.

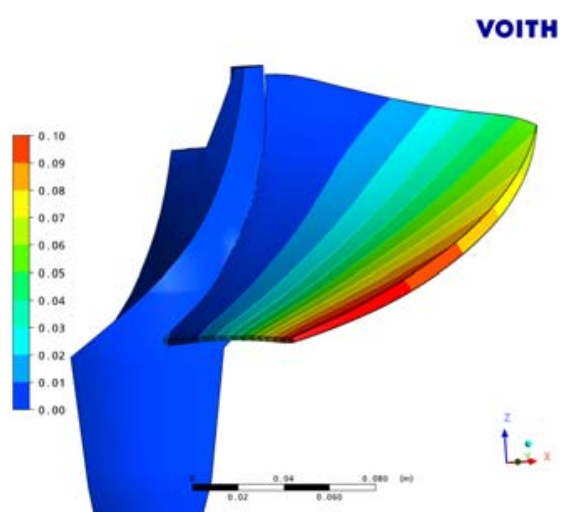

F2

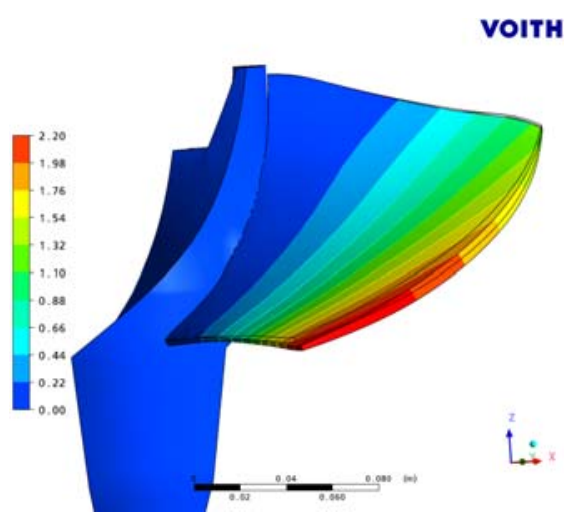

F4

Fig. 4 Normalized deflection of the turbine blade for the nominal blade stiffness (F2, left) and the least stiff one (F4, right).

In order to highlight the influence of the blade deformation on the fluid field and thereby also on the pressure loading of the turbine blade Fig. 5 shows pressure contours on the pressure and suction side for the undeformed (F1) and the maximal deformed blade (F4), both from the fully coupled FSI simulations. There is a significant impact noticeable especially around the leading edge of the blade on both sides, the pressure and suction side. This is a result of the modified flow incidence angle due to the blade deformation of approximately $90 \%$ of the trailing edge thickness, as seen in Fig. 4. 

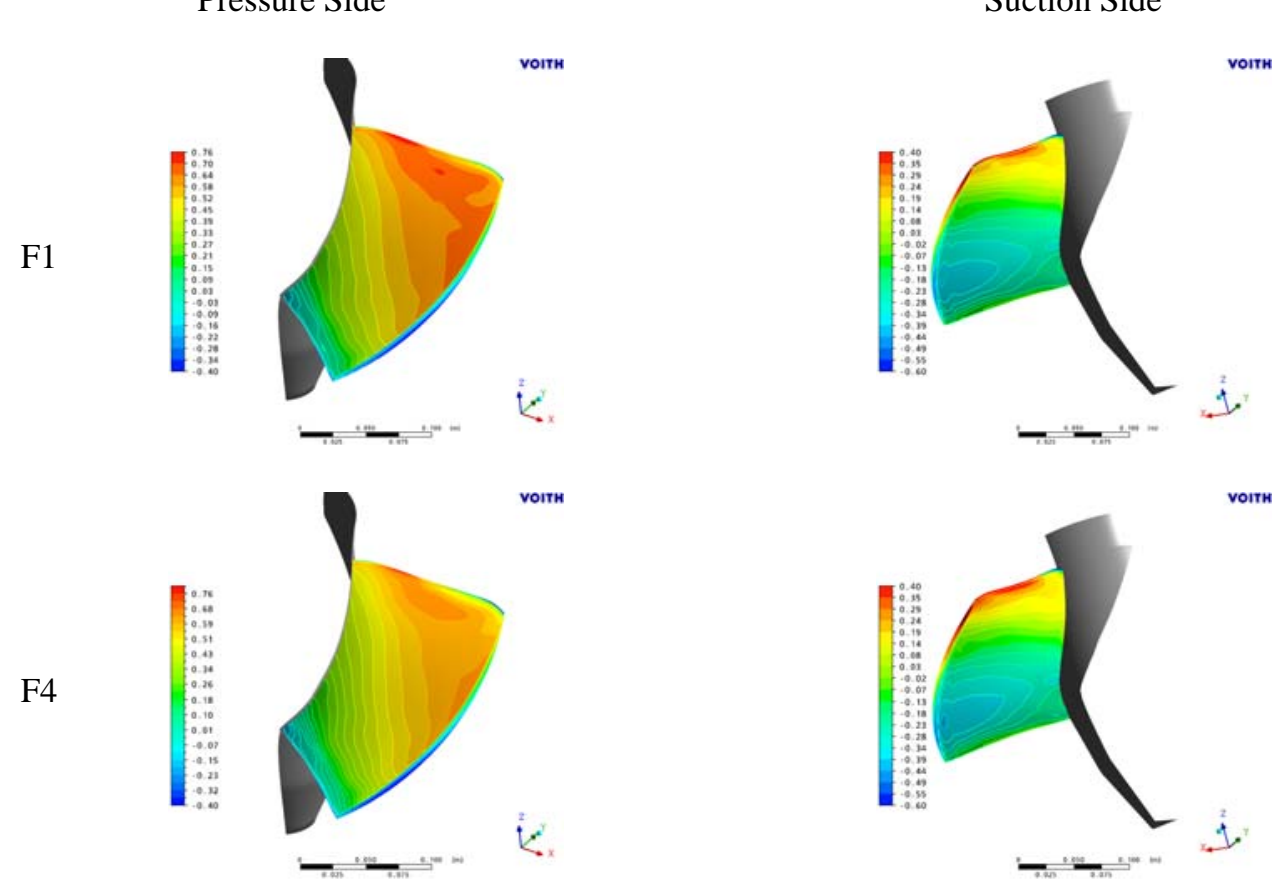

Fig. 5 Pressure distributions for the undeformed (F1) and maximal deformed blade (F4).

Finally, the diagram presented in Fig. 6 summarizes the normalized blade deflections at the trailing edge tip of the blade for the different FSI calculations performed. The results are consistent in the sense, that blade deflection is inversely proportional to blade stiffness. Additionally, a refined structural model (F4b) did not yield a different answer as the standard model (F4) which indicates that the structural solution of the FSI runs is not dependent on the numerical grid. In the future such a grid refinement study will be performed for the fluid field as well, but the mesh sizes are standard setups of Voith Hydro and therefore known to yield grid independent CFD results. Also noticeable is a slightly larger deflection for the setup without a blade tip gap. This can be expected since the pressure acts on a larger blade surface yielding a larger pressure load and runner shaft torque which will be demonstrated later. Finally, the deflection of the three nominal stiffness setups (F2, G2, and C2a) are very similar, though the actual deflection of the setup without the gap is larger by $0.2 \%$ of the trailing edge thickness if one compares the actual numbers.

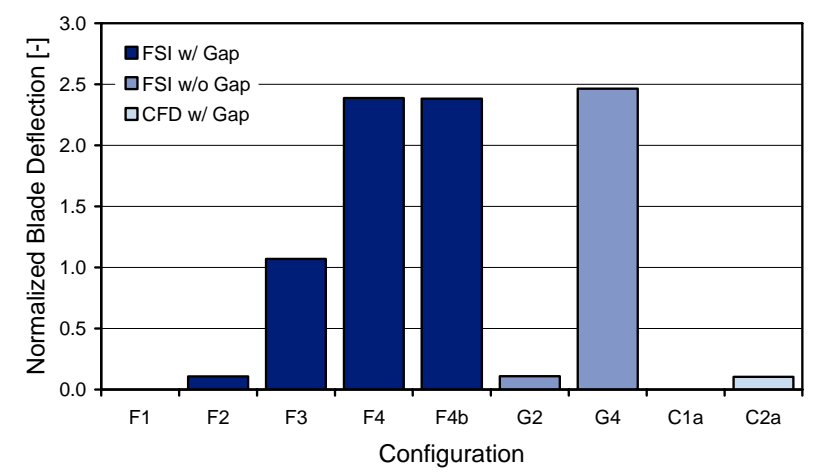

Fig. 6 Comparison of normalized blade deflections for different configurations.

\subsection{Velocity Profiles}

As mentioned before, the Consortium on Hydraulic Machines at the LAMH performed extensive velocity measurements on the AxialT turbine [6]. In Fig. 7 the measurement locations downstream of the runner are visualized. These measurements have been performed by laser doppler velocimetry (LDV) as well as stereoscopic particle image velocimetry (PIV). In the following, simulated profiles are compared to the LDV measurements at the location closest to the runner trailing edge, namely elevation A. The error bars included for the experimental data indicate the standard deviation of the results over time. This includes not only turbulent fluctuations, but also coherent structures like the blade tip vortex or other blade passing effects. All velocity profiles have been normalized using the average axial velocity at the selected cross section calculated by dividing the volume flow rate by the cross sectional area. The simulation results have been obtained by a mass flow averaged circumferential average. 


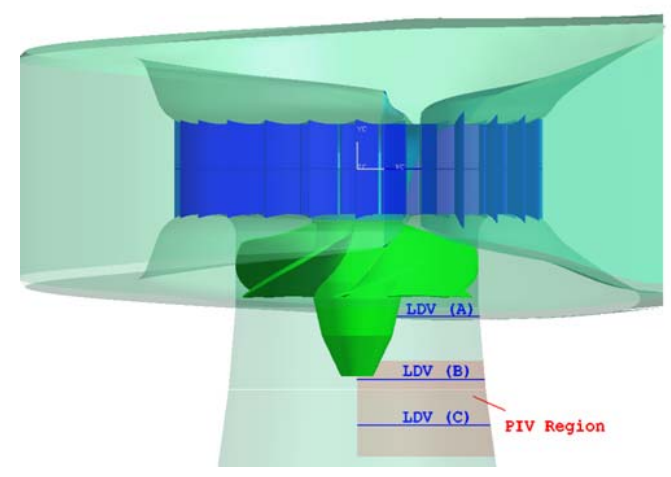

Fig. 7 Measurement locations for velocity profiles with LDV and stereoscopic PIV.

The first comparison in Fig. 8 is used to show the generally good agreement between the simulations of the undeformed blade (F1, C1a) and the LDV measurements similar to the more detailed analysis presented previously [1]. While the normalized axial velocity profiles completely fall on top of each other there is a very small difference in the normalized tangential velocity profile noticeable towards the periphery. Additionally, the deviation from the experimental measurements is evident in this location (see also zoom box). In this region the gap has a strong influence on the results. In the simulations, the nominal gap has been used which resembles an average gap size over all six blades of the AxialT turbine. The small difference between the two simulations is related to differences in the mesh especially in this region as the mesh was adapted and optimized for the use in the FSI calculations in order to allow for larger deformations of the blade. The pure CFD calculation (C1a) on the other hand was taken from the initial work [1].

The second comparison in Fig. 9 includes the calculation results for the nominal stiffness for both, the fully two-way coupled FSI runs (F1, F2) and the manually coupled CFD only simulations (C1a, C2a). Again, the axial velocity cannot be used to distinguish between the different configurations. The normalized tangential velocity on the other hand shows some influence for the FSI calculations while the CFD simulations result in almost identical results. Looking at the actual numbers, the simulations C1a and C2a differ by roughly 1\% between the two simulations which is not visible in the figure. This difference between the FSI and CFD runs might be one possible explanation to the inconsistencies that are encountered and described for the runner shaft torque later in this paper. In general, from the velocity profile results for the undeformed and the nominal stiffness turbine blade it is not to be expected, that there is a noticeable impact on turbine performance.
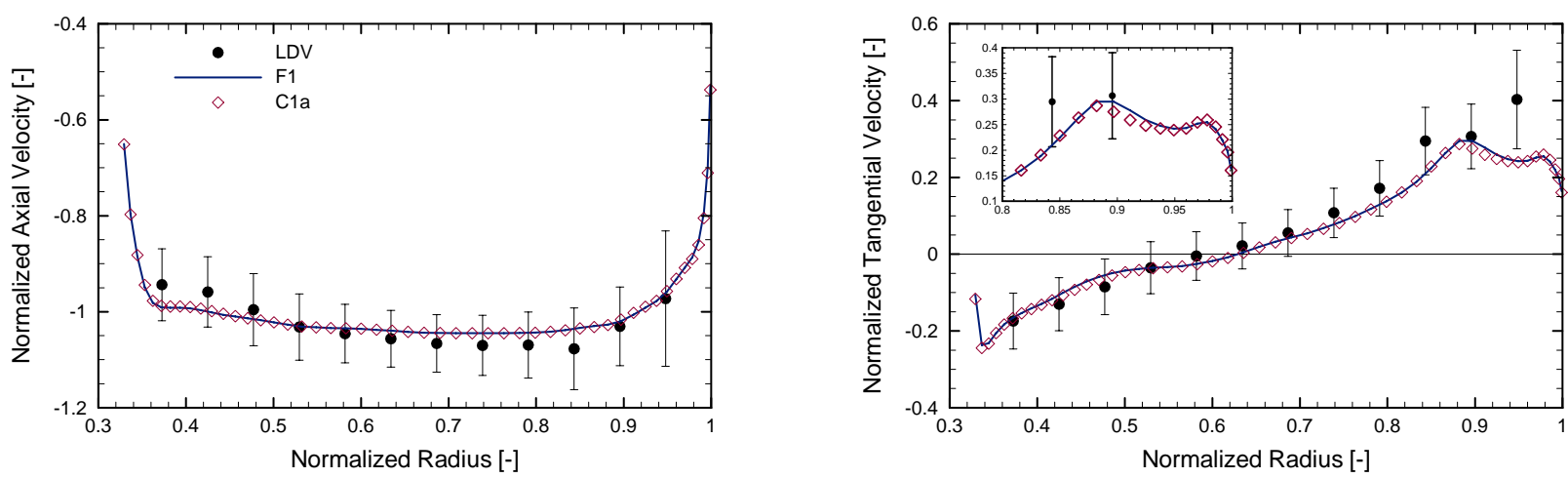

Fig. 8 Comparison of velocity profiles for the undeformed blade obtained by fully two-way coupled FSI (F1) and CFD only (C1a).
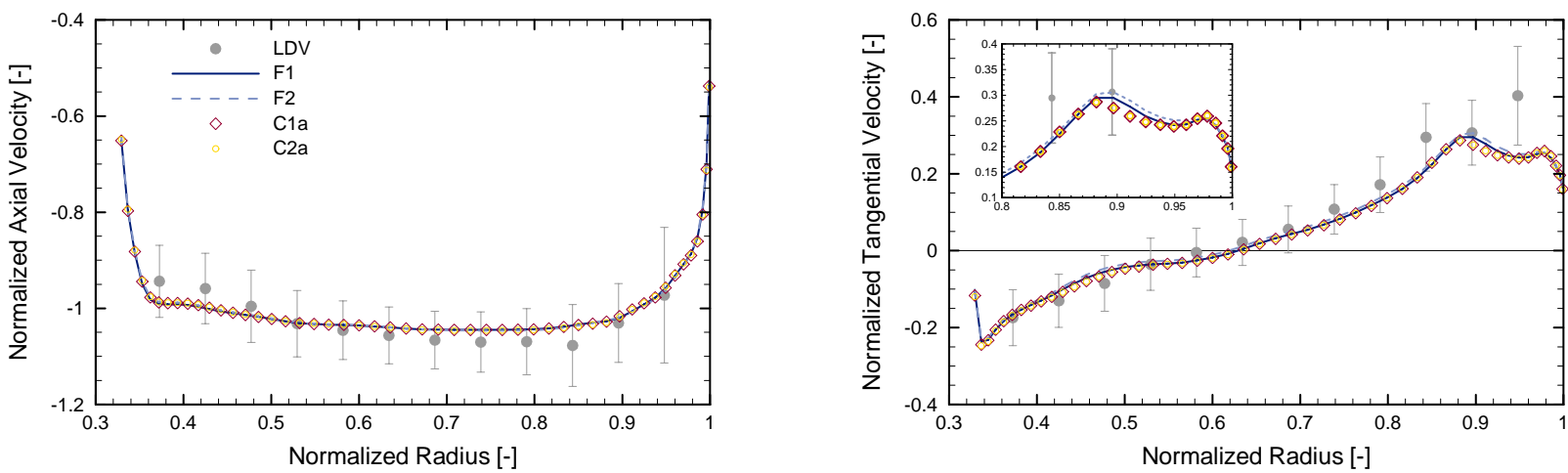

Fig. 9 Comparison of velocity profiles for the undeformed (F1, C1a) and nominal stiffness (F2, C2a) simulations.

A much stronger influence on the velocity discharge profile can be seen for lower blade stiffness. The normalized velocity profiles are presented in Fig. 10 for the various two-way coupled FSI simulations (F1, .., F4). Now, the axial velocity distribution 
also exhibits a modification as the blade stiffness is reduced. While it reduces towards the smaller radius at the hub it increases towards the periphery at the large radius. Interestingly enough it seems to match better with the experimental findings. Also, the tangential velocity exhibits a clear trend to larger values with reduced stiffness. The values are shifted by an almost constant offset. As a result more swirl is present at the outlet of the runner which in turn lowers the performance of the turbine assuming a constant inlet swirl into the runner. Furthermore, the inflection point from positive to negative swirl shifts from a radial position of approximately 0.63 to 0.59 . All these effects on the velocity profile will have a significant impact on the draft tube performance which is of high importance in low head applications. To quantify this influence the draft tube needs to be included in the simulations.
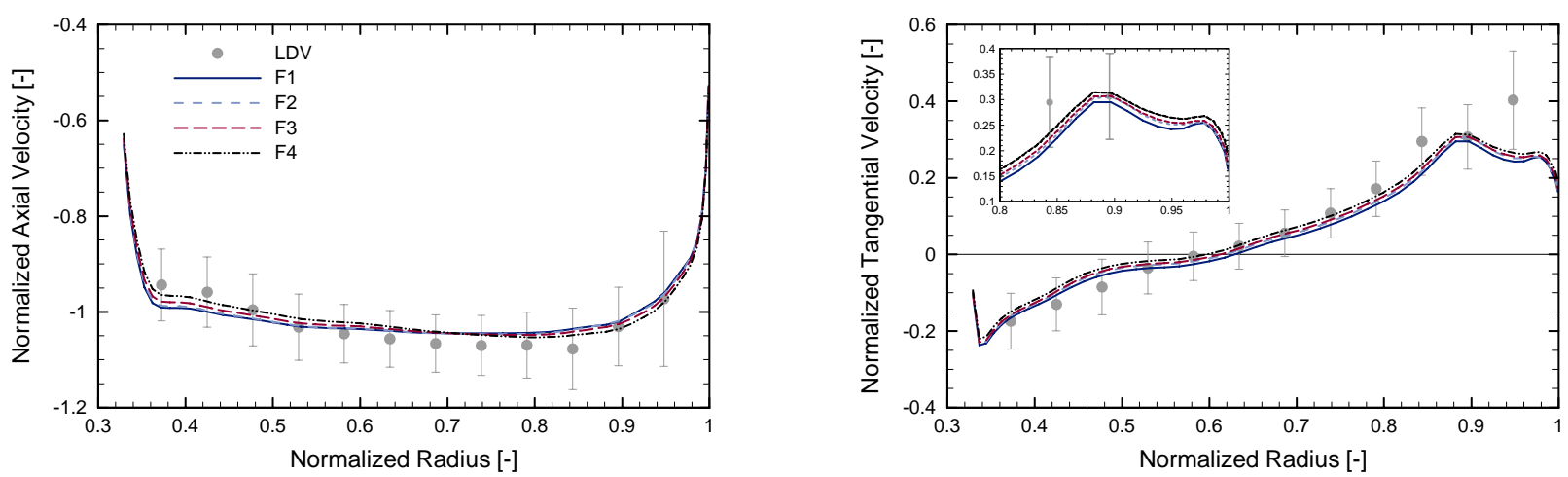

Fig. 10 Comparison of velocity profiles for the two-way coupled FSI calculations with decreasing stiffness (F1, ..., F4).

In a final velocity profile comparison the configurations with and without the gap for the nominal and the smallest blade stiffness (F2, F4 and G2, G4) are provided in Fig. 11. These comparisons are similar to the investigations presented in [1]. As expected, there is a general difference between the simulation with and without the peripheral gap for both, the axial as well as the tangential velocity component. It is known that this difference has a strong influence on the draft tube performance, since especially near the wall of the diffuser an accurate representation of the real flow conditions is required to predict the onset of flow separation [7]. In general, the calculations including the gap match better with the experimental results. Furthermore, the influence of the reduced stiffness seems to be very similar for both types of calculations and was described already in the context of the previous figure.
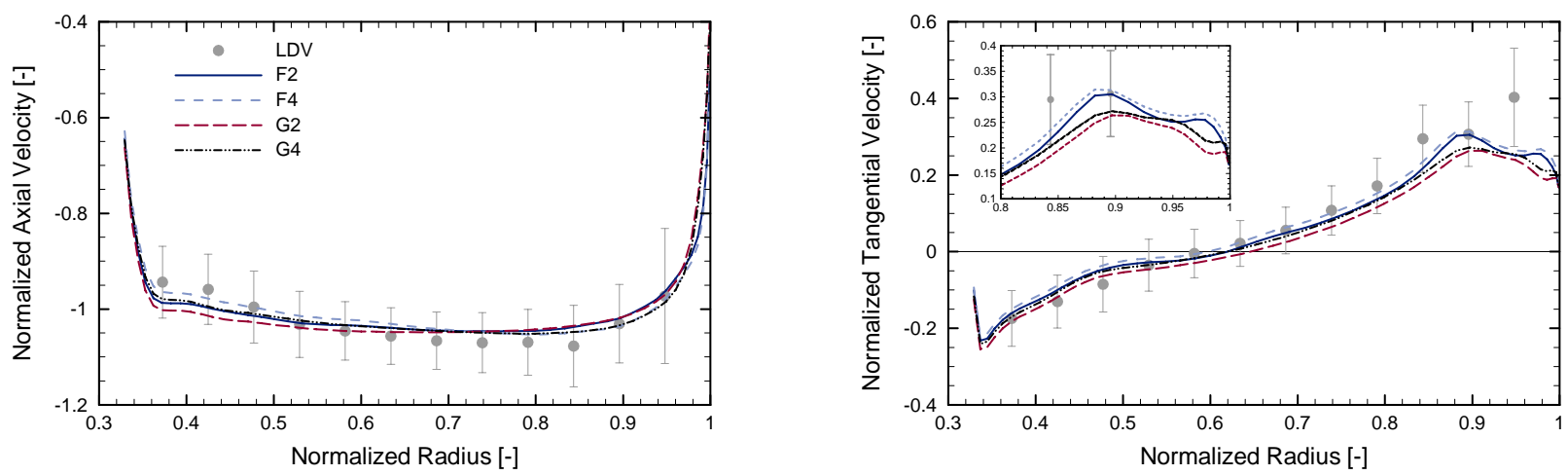

Fig. 11 Comparison of velocity profiles for simulations with (F2, F4) and without the tip gap (G2, G4) for two different stiffness.

\subsection{Tip clearance vortex}

The laser measurements of the Consortium on Hydraulic Machines at the LAMH has provided the opportunity to visualize the velocity not only as a $2 \mathrm{D}$ velocity profile with circumferential averaging as discussed above, but also as a $2 \mathrm{D}$ plot with local velocity values on the evaluation planes. The same evaluation planes were used for the CFD post processing, so the results of the two approaches could be compared and validated.

Besides the direct comparison of the two methods the influence of the tip clearance vortex can be analyzed. The size of the tip clearance is a compromise between mechanical needs and hydraulic influence. The vortex results from the leakage through the gap from pressure to suction side. The gap has three main effects on the hydraulic behavior. At first the range of leakage influences the efficiency of the turbine, second the vortex effects the cavitation behavior of the runner and third it stabilizes the draft tube flow.

So the analysis of the velocity plots concentrates not only on the comparison of the two different investigation methods, but also studies the influence by the blade deformation on the gap flow. All velocity plots have been normalized using the average axial velocity at the selected cross section calculated by dividing the volume flow rate by the cross sectional area. The simulation results have been obtained by a mass flow averaged circumferential average.

The velocity plots of the axial component in Fig. 12 on evaluation plane A (location compare Fig. 7) show an inhomogeneous distribution in radial and tangential direction. The inhomogeneity is a result of the design of the runner and caused by the layout of the flow channel between two blades. According to the clearance between the two blades and variation of the blade length at 
different radial positions the flow distribution changes. The tangential distribution of the axial velocity is dominated by the position of the blades. In the area of the trailing edge of the blades the wakes are visible with low axial velocity. On the pressure side an area with high velocity appears which decreases towards the middle of the flow channel and increases again near the suction side of the blade, but with lower maximum speed. Also the radial distribution of the axial velocity varies. In the area of the pressure side the axial velocity has a maximum on the shroud side and decreases in negative radial direction. The same behavior can be noticed in the area of the suction side. The velocity in the middle of the flow channel is more uniform with a small peak half way in radial direction. This qualitative behavior is the same for each of the configurations shown in Fig. 12. A very good quantitative agreement has been achieved for the laser measurements and the model with nominal stiffness (F2). A larger deformation of the blade (F4) leads to a higher maximum axial velocity at the shroud near the pressure side. At the same time the velocity distribution in the middle of the channel shows a more uniform velocity profile. Also the tip clearance has a visible effect on the axial velocity distribution. The same runner as F2 without gap (G2) is characterized by a more uniform axial velocity distribution than F2 in pressure side area. The other areas are similar to F2.

Besides the global velocity distribution the tip clearance vortex can also be observed in the axial velocity plots. Vortices have high circumferential velocities and therefore a low axial velocity. So the position of the gap vortex can be seen as area with low axial velocity. At the shroud side of the results of LDV, F2 and F4 such an area is visible between pressure and suction side of the blade. G2 without clearance modeling does not show such an area of low velocity.

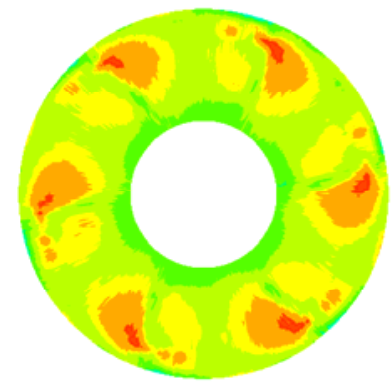

a) LDV

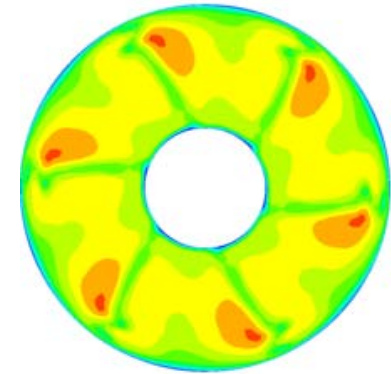

b) F2

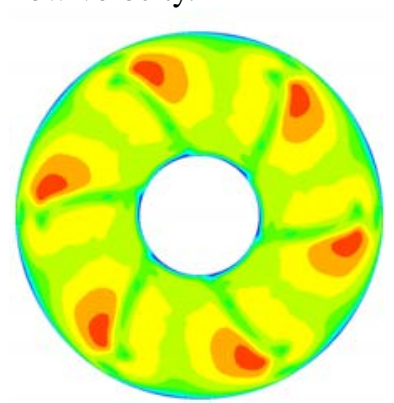

c) F4

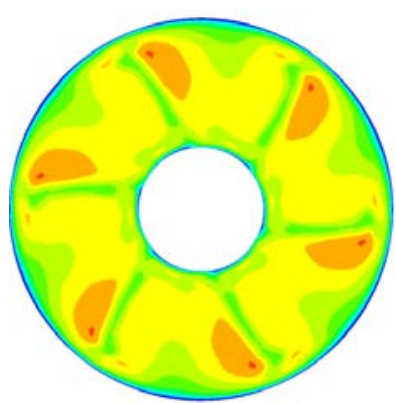

d) G2

Fig. 12 Visualization of deflection of runner blade for different configurations

The tip clearance vortex can also be visualized by looking at the tangential velocity information (Fig. 13).The models with gap (LDV, F2, F4) show two velocity peaks at each blade. The larger velocity peak which is more significant describes the upper channel vortex. Between this vortex and the shroud a small tangential velocity peak is visible. This spot describes the vortex which is caused by the gap. The LDV measurement shows very good agreement for the gap area with the results of F2. The deflection of the blade shows an influence on the vortex area, because the main deformation of the blade appears at this location. But this influence is small and the change is not of significant size. The investigation of the model without gap (G2) confirms the theory of the gap vortex, because the second small velocity peak disappears within this simulation. The second phenomenon is the reduction of the maximum velocity of the blade vortex. So the gap has not only influence on the gap vortex, but also increases the strength of the channel vortex.

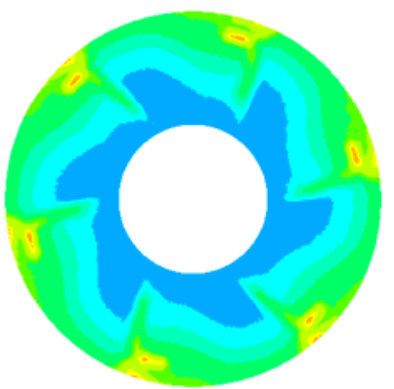

a) LDV

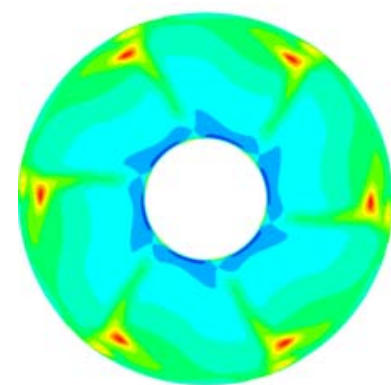

b) F2

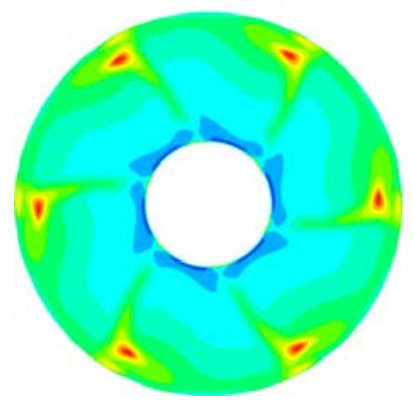

c) F4

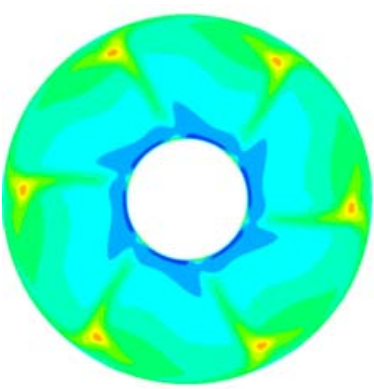

d) G2

Fig. 13 Visualization of deflection of runner blade for different configurations

\subsection{Runner Shaft Torque}

As a measure for the performance of the turbine the runner shaft torque around the rotational axis of the turbine has been chosen. The normalization is performed with the shaft torque of the fully two-way coupled FSI calculation employing the nominal stiffness (F2). In Fig. 14(a) all previously discussed calculations are compared. As expected, the reduced stiffness lowers the shaft torque for the AxialT turbine configuration as can be seen for the configurations F1 through F4. It is surprising though, that there is a difference of $1 \%$ between the undeformed case F1 and the nominal stiffness case F2. Considering the small deflection for the F2 case as shown in Fig. 4, one would expect almost no difference in shaft torque. This expectation is in line with the findings for the pure CFD simulations C1a and C2a. These two simulations do not differ noticeably in shaft torque and are at the same level as the undeformed FSI run F1. Again, the refined structural mesh (F4b) results in identical results as the standard mesh (F4) which 
was to be expected since the deflections are identical as well. The two simulations without the gap at the blade tip (G2, G4) exhibit a 3\% higher blade torque for both setups when compared to the similar setup with gap.

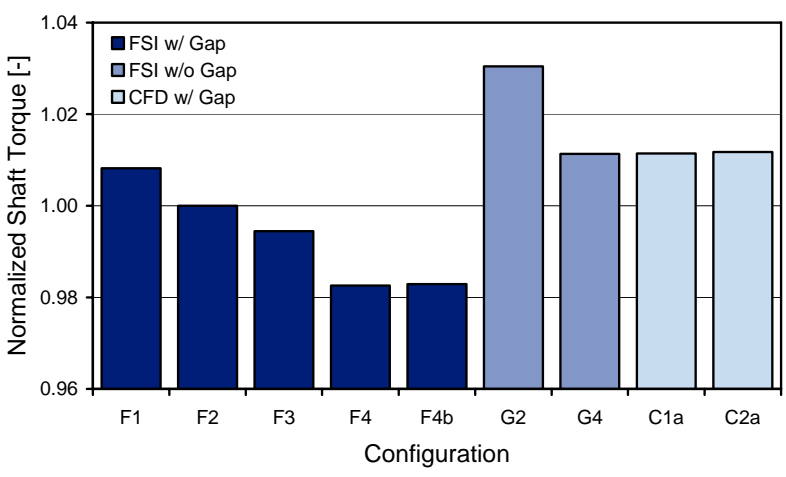

(a)

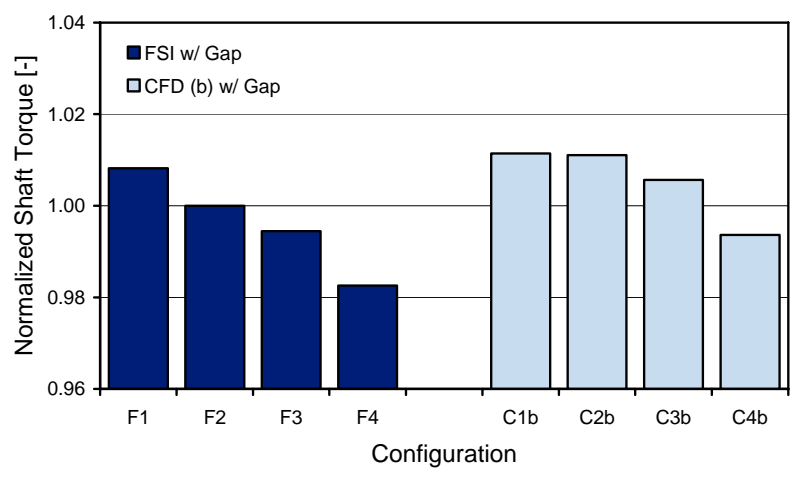

(b)

Fig. 14 Comparison of normalized runner torque for different configurations (a) and alternative CFD calculations (b).

In order to investigate the inconsistency of the shaft torque results further the second set of CFD calculations has been performed (C1b, ..., C4b). These calculations are based on the geometry and mesh of the corresponding converged two-way coupled FSI calculations (F1, ..., F4). Since the fluid field is solved at the end of the multi-field procedure by ANSYS, the results should reproduce the FSI results. Unfortunately, as one can see in Fig. 14(b), the results turn out to be different. While the undeformed blade C1b still yields identical results as the FSI calculation F1, the previously observed drop of $1 \%$ in shaft torque to the nominal stiffness for F1 to F2 is no longer present for C1b to C2b. All further simulations at lower stiffness have an offset of about $1 \%$ compared to the FSI calculations. In general, these shaft torques look like the initially expected results from the setup and design of this study, as well as expected from the deflections shown in Fig. 6.

More investigations have been done in close contact with ANSYS CFX support and the FSI results could be adjusted to the pure CFD results. So the rerun of the fully two-way coupled simulations lead to the same results as the pure CFD runs as shown in Fig. 14(b) for C1b to C4b. Finally the influence of the blade deflection on the performance of the turbine between a rigid runner (C1b) and a runner with original material properties (C2b) is not ascertainable and in a very small range. But the theoretical approach with decreasing stiffness of the material shows a significant tendency to lower performance of the turbine.

\section{Conclusion}

In order to predict the performance of hydraulic machines for low head applications an accurate representation of the correct physical phenomena is essential. Therefore the interaction of the fluid with the turbine blade structure will become more and more important for high performance low head turbine designs since thinner blades and less material tend to decrease the stiffness of the structure. As a consequence larger blade deflections are encountered and the hydraulic shape is modified possibly in an unfavorable manner. In the past, multi-field simulations have been applied to generic test cases easy to investigate experimentally in a laboratory or simplified to improve the stability of the coupled numerical solution. The presented work analyzed these phenomena on an actual model turbine blade using engineering tools which are applied in the blade design process at Voith Hydro. For the actual AxialT model configuration, no major influence was detected when comparing the undeformed blade to the deflected blade. Once the blade stiffness was reduced, a significant influence was observed. The reduction in blade stiffness was achieved by varying Young's Modulus for the propeller turbine blade. Applying modern simulation software it is possible to simulate the fully two-way coupled fluid-structure interaction problem and demonstrate the influence on the hydraulic performance of the model turbine. Inconsistencies in the results have been investigated and resolved. Here, Voith Hydro has worked closely with the software manufacturer ANSYS in order to identify the problems and come to a solution for the tool.

\section{Acknowledgments}

The authors would like to thank the participants on the Consortium on Hydraulic Machines for their support and contribution to this research project: Alstom Hydro Canada, Andritz Hydro, Edelca, Hydro-Quebec, Laval University, NRCan, Voith Hydro. Our gratitude goes as well to the Canadian Natural Science and Engineering Research Council who provided funding for this research. Without these contributions no high quality velocity measurements would have been available for this turbine to compare to.

\section{References}

[1] Cunha, F., Qian, R., Flemming, F., Chen, L. and Riedel, N., 2009, "Detailed Analysis of Flow Features in Propeller Turbines,” Waterpower XVI, Spokane, WA, USA.

[2] Hübner, B., Seidel, U. and Scherer, T., 2006, "Fluid-Structure Interaction with ANSYS and CFX - Investigation of Convergence Properties of Partitioned Solutions to Hydroelastic Systems," $24^{\text {th }}$ CADFEM Users' Meeting, Stuttgart, Germany.

[3] Münch, C., Ausoni, P., Braun, O., Farhat, M. and Avellan, F., 2008, "Hydro Elastic Behavior of Vibrating Blades,” $24^{\text {th }}$ IAHR Symp. on Hyd. Machinery and Systems, Foz do Iguassu, Brazil. 
[4] Hübner, B., Seidel, U. and Roth, S., 2010, “Application of FLuid-Structure Coupling to Predict the Dynamic Behavior of Turbine Components,” $25^{\text {th }}$ Symp. on Hyd. Machinery and Systems, Timisoara, Romania.

[5] ANSYS CFX Release 11.0, 1996-2006, Documentation, ANSYS Europe, Ltd.

[6] Gagnon, J.M., Illiescu, M., Ciocan, G.D. and Deschênes, C., 2008, "Experimental Investigation of Runner Outlet Flow in Axial Turbine with LDV and Stereoscopic PIV,” $24^{\text {th }}$ IAHR Symp. on Hyd. Machinery and Systems, Foz do Iguassu, Brazil.

[7] Hübner, B., Aschenbrenner, T., Kächele, T., Suzuki, R., 2008, “Flow Prediction in Bulb Turbines,” $24^{\text {th }}$ IAHR Symp. on Hyd. Machinery and Systems, Foz do Iguassu, Brazil. 